ISSN 2075-9827 e-ISSN 2313-0210

Carpathian Math. Publ. 2018, 10 (2), 273-287

doi:10.15330/cmp.10.2.273-287

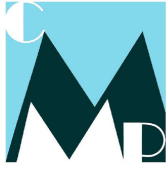

http://www.journals.pu.if.ua/index.php/cmp

Карпатські матем. публ. 2018, Т.10, №2, С.273-287

BURTNYAK I.V., MALYTSKA H.P.

\title{
APPLICATION OF THE SPECTRAL THEORY AND PERTURBATION THEORY TO THE STUDY OF ORNSTEIN-UHLENBECK PROCESSES
}

\begin{abstract}
The theoretical bases of this paper are the theory of spectral analysis and the theory of singular and regular perturbations. We obtain an approximate price of Ornstein-Uhlenbeck double barrier options with multidimensional stochastic diffusion as expansion in eigenfunctions using infinitesimal generators of a $(l+r+1)$-dimensional diffusion in Hilbert spaces. The theorem of accuracy estimation of options prices approximation is established. We also obtain explicit formulas for derivatives price based on the expansion in eigenfunctions and eigenvalues of self-adjoint operators using boundary value problems for singular and regular perturbations.

Key words and phrases: spectral theory, singular perturbation theory, regular perturbation theory, Sturm-Liouville theory, infinitesimal generator, multidimensional diffusion.
\end{abstract}

Vasyl Stefanyk Precarpathian National University, 57 Shevchenka str., 76018, Ivano-Frankivsk, Ukraine

E-mail: bvanya@meta.ua (Burtnyak I.V.)

\section{INTRODUCTION}

In 1956 McKean H.P. constructed a spectral presentation for general one-dimensional diffusion [1]. Since then, spectral theory has become an important instrument for the analysis of financial diffusion models, as investigation of expansion in eigenfunctions of linear operators. Many problems concerning derivatives estimation are solved using methods of spectral theory; as a result it is widely used in financial mathematics.

Spectral theory has been extensively applied by many scientists, namely, to forecast call option price [2], to find interest rates on securities [3] and model volatility of financial assets. Both spectral theory and stochastic volatility models have become an indispensable tool in mathematics of finance [4], due to the fact that prices of double barrier options are subjected to the Brownian motion and are correlated with volatility [5]. Therefore, it is employed in an investigation of stochastic volatility, in particular the asset volatility, which is the basis of controlled and nonlocal diffusion [6]. Applying methods of spectral theory, theories of singular and regular perturbations, we can obtain approximate price of Ornstein-Uhlenbeck double barrier options with multidimensional volatility, as expansion in eigenfunctions using infinitesimal generators of a $(l+m+1)$-dimensional diffusion, $l \geq 1, r \geq 1, l \in \mathbb{N}, r \in \mathbb{N}$, i.e. the diffusion depends on one local variable, $l$-dimensional fast variable and $n$-dimensional slow variable. This paper develops the following researches [7-9], in [9] it is considered the case $l=1$ and $m=1$.

The purpose of the article is to elaborate algorithms for evaluating approximate price of double barrier options and to find explicit formulas for derivatives estimation as expansion in 
eigenfunctions and eigenvalues of self-adjoint operators using boundary problems for singular and regular perturbations. The theorem of accuracy estimation of options prices approximation is established.

\section{RESUlts}

Let $(Q, F, P)$ be the probability space that supports a correlated Brownian motion $\left(\mathrm{W}^{x}, \mathrm{~W}^{y_{1}}, \ldots, \mathrm{W}^{y_{l}}, \mathrm{~W}^{z_{1}}, \ldots, \mathrm{W}^{z_{r}}\right)$ and an exponential random variable $\varepsilon \sim \exp (1)$, which is independent of $\left(\mathrm{W}^{x}, \mathrm{~W}^{y_{1}}, \ldots, \mathrm{W}^{y_{l}}, \mathrm{~W}^{z_{1}}, \ldots, \mathrm{W}^{z_{r}}\right)$. We will assume that the economy with $(l+r+1)$ factors is described by the homogeneous time and continuous Markov process $X=\left(X, Y_{1}, \ldots, Y_{l}, Z_{1}, \ldots, Z_{n}\right)$, which is defined in some state space $E=I \times R^{l} \times R^{r}$, where $\left(Y_{1}, \ldots, Y_{l}\right) \in \mathrm{R}^{l},\left(Z_{1}, \ldots, Z_{r}\right) \in \mathrm{R}^{r}, I$ is the interval at $R$ with points $e_{1}$ and $e_{2}$, such that $-\infty<$ $e_{1}<e_{2}<\infty$. We assume that $X$ has the beginning at $E$ and instantly disappears once $X$ goes beyond $I$. In particular, the dynamics of $X$ with physical measure $\mathbb{P}$ is as follows:

$$
\boldsymbol{X}_{t}= \begin{cases}\left(X_{t}, Y_{1 t}, \ldots, Y_{l t}, Z_{1 t}, \ldots, Z_{r t}\right), & \tau_{I}>t \\ \Delta, & \tau_{I} \leq t\end{cases}
$$

$\tau_{I}=\inf \left\{t>0: X_{t} \notin I\right\}$, where $\left(X, Y_{1}, \ldots, Y_{l}, Z_{1}, \ldots, Z_{r}\right)$ are set

$$
\begin{cases}d X_{t}=v\left(X_{t}\right) d t+a\left(X_{t}\right) f\left(Y_{1 t}, \ldots, Y_{l t}, Z_{1 t}, \ldots, Z_{r t}\right) d W_{t}^{x}, & \\ d Y_{j t}=\frac{1}{\varepsilon_{j}} \alpha_{j}\left(Y_{j t}\right) d t+\frac{1}{\sqrt{\varepsilon_{j}}} \beta_{j}\left(Y_{j t}\right) d W_{t}^{y_{j}}, & j=\overline{1, l}, \\ d Z_{i t}=\delta_{i} c_{i}\left(Z_{i t}\right) d t+\sqrt{\delta_{i}} g_{i}\left(Z_{i t}\right) d W_{t}^{z_{i},} & i=\overline{1, r}, \\ d\left(W^{x}, W^{y_{j}}\right)_{t}=\rho_{x y_{j}} d t, & j=\overline{1, l} \\ d\left(W^{x}, W^{z_{i}}\right)_{t}=\rho_{x z_{i}} d t, & i=\overline{1, r}, \\ d\left(W^{y_{j}}, W^{z_{i}}\right)_{t}=\rho_{y_{j} z_{i}} d t, & j=\overline{1, l}, i=\overline{1, r} \\ d\left(W^{y_{j}}, W^{y_{r}}\right)_{t}=\rho_{y_{j} y_{s}} d t, & j=\overline{1, l}, s=\overline{1, l} \\ d\left(W^{z_{i}}, W^{z_{k}}\right)_{t}=\rho_{z_{i} z_{k}} d t, & i=\overline{1, n, k}=\overline{1, r}, \\ \left(X, Y_{1}, \ldots, Y_{l}, Z_{1}, \ldots, Z_{r}\right)=\left(x, y_{1}, \ldots, y_{l}, z_{1}, \ldots, z_{r}\right) \in E, & \end{cases}
$$

where $\rho_{y_{j} y_{s}}=0, j \neq r, \rho_{z_{i} z_{k}}=0, i \neq k, \rho_{x y_{j}}, \rho_{x z_{i}}, \rho_{y_{j} z_{i}}$ meet the conditions ||$\rho_{x y_{j}}|\leq 1,| \rho_{x z_{i}} \mid \leq 1$, $\left|\rho_{y_{j}} z_{i}\right| \leq 1$, and correlation matrices of the form

$$
\left(\begin{array}{ccc}
1 & \rho_{x y_{j}} & \rho_{x z_{i}} \\
\rho_{y_{j} x} & 1 & \rho_{y_{j} z_{i}} \\
\rho_{z_{i} x} & \rho_{z_{i} y_{j}} & 1
\end{array}\right)
$$

semipositively defined, that is $1+2 \rho_{x y_{j}} \rho_{x z_{i}} \rho_{y_{j} z_{i}}-\rho_{x y_{j}}{ }^{2}-\rho_{x z_{i}}{ }^{2}-\rho_{y_{j} z_{i}}{ }^{2} \geq 0, j=\overline{1, l}, i=\overline{1, r}$. Process $X$ may represent many economic phenomena and processes.

For example, the reserve size, the index price and reliable short-term interest rates, etc. Even more broadly, $X$ is an external factor that characterizes the value of any of the abovementioned processes. Physical measure $\mathbb{P}$ of process $X$ is understood as the process $X$, which has an instant drift $v\left(X_{t}\right)$ and stochastic volatility $a\left(X_{t}\right) f\left(Y_{1 t}, \ldots, Y_{l t}, Z_{1 t}, \ldots, Z_{n t}\right)>0$, which 
contains both components: local $a\left(X_{t}\right)$ and nonlocal $f\left(Y_{1 t}, \ldots, Y_{l t}, Z_{1 t}, \ldots, Z_{n t}\right)$. Note that infinitesimal generators for $Y_{j}$ and $Z_{i}$ have the form

$$
\mathfrak{L}_{Y_{j}}^{\varepsilon_{j}}=\frac{1}{\varepsilon_{j}}\left(\frac{1}{2} \beta_{j}^{2}\left(y_{j}\right) \partial_{y_{j} y_{j}}^{2}+\alpha_{j}\left(y_{j}\right) \partial_{y_{j}}\right), \quad \mathfrak{L}_{Z_{i}}^{\delta_{i}}=\delta_{i}\left(\frac{1}{2} g_{i}{ }^{2}\left(z_{i}\right) \partial_{z_{i} z_{i}}^{2}+c_{i}\left(z_{i}\right) \partial_{z_{i}}\right), \quad \forall i, j,
$$

and are characterized by the measures $\frac{1}{\varepsilon_{j}}$ and $\delta_{i}$, respectively. Thus, $Y_{1}, \ldots, Y_{l}$ and $Z_{1}, \ldots, Z_{n}$ have an internal time scale $\varepsilon_{j}>0$ and $\frac{1}{\delta_{i}}>0$. We consider $\varepsilon_{j}<<1$ and $\delta_{i}<<1$, so that the internal time scale $Y_{j}$ is small, and the internal time scale $Z_{i}$ is large. Consequently, $Y_{j}, j=\overline{1, l}$, are fast variables, and $Z_{i}, i=\overline{1, n}$, are slow variables. Note that $\mathfrak{L}_{Y_{j}}^{\mathcal{\varepsilon}_{j}}$ and $\mathfrak{L}_{Z_{i}}^{\delta_{i}}$ have the form

$$
\mathfrak{L}=\frac{1}{2} a^{2}(x) \partial_{x x}^{2}+b(x) \partial_{x}-k(x), x \in\left(e_{1}, e_{2}\right), c ̧ k(x)=0,
$$

for all $x \in I$, are always self-adjoint in the Hilbert space $H=L^{2}(I, m)$, where $I \in R$ is the interval with the points $e_{1}$ and $e_{2}$ and $m$ is the diffusion density rate. Note,

$\operatorname{Dom}(\mathfrak{L})=\left\{f \in L^{2}(I, m): f, \partial_{x} f \in A C_{\text {loc }}(I), \mathfrak{L} f \in L^{2}(I, m), B C s\right.$ on $e_{1}$ and $\left.e_{2}\right\}$, where $A C_{\text {loc }}(I)$ is the space of functions which are absolutely continuous on each compact subinterval $I$ (see [9]). The boundary conditions for $e_{1}$ and $e_{2}$ are applied on the output, input, and regular bounds.

We will evaluate the derivatives with payoff at time $t>0$, which may depend on the trajectory of $X$. In particular, we will consider the forms of payoff: Payoff $=H\left(X_{t}\right) \mathbb{I}_{(\tau>t)}$, where $\tau$ is a random moment of time during which there is a failure to make a payment of premium. Since we are interested in the derivatives estimation, we must determine the dynamics $\left(X, Y_{1}, \ldots, Y_{l}, Z_{1}, \ldots, Z_{r}\right)$ under the evaluation of the degree of neutral risk, which we denote as $\widetilde{\mathbb{P}}$. We have the following dynamics

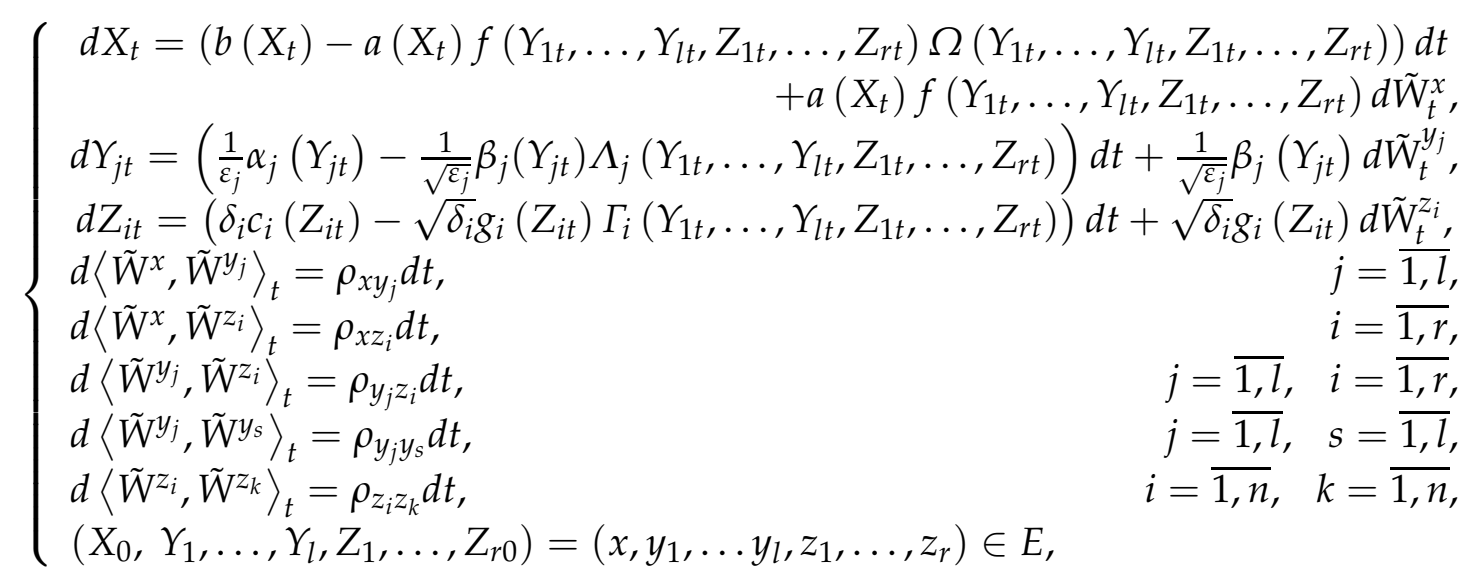

where $\rho_{y_{j} y_{s}}=0, j \neq s, \rho_{z_{i} z_{k}}=0, i \neq k$, and

$$
\begin{gathered}
d \tilde{W}_{t}^{x}:=d W_{t}^{x}+\left(\frac{v\left(X_{t}\right)-b\left(X_{t}\right)}{a\left(X_{t}\right) f\left(Y_{1 t}, \ldots, Y_{l t}, Z_{1 t}, \ldots, Z_{n t}\right)}+\Omega\left(Y_{1 t}, \ldots, Y_{l t}, Z_{1 t}, \ldots, Z_{r t}\right)\right) d t \\
d \tilde{W}_{t}^{y_{j}}:=d W_{t}^{y_{j}}+\Lambda_{j}\left(Y_{1 t}, \ldots, Y_{l t}, Z_{1 t}, \ldots, Z_{r t}\right) d t \\
d \tilde{W}_{t}^{z_{i}}:=d W_{t}^{z_{i}}+\Gamma_{i}\left(Y_{1 t}, \ldots, Y_{l t}, Z_{1 t}, \ldots, Z_{r t}\right) d t .
\end{gathered}
$$

We establish such conditions so that the system (1) has the only strong solution.

Random time $\tau$ is the time of the derivative asset. In our case, default can occur in one of two ways: 
1) when $X$ fall outside the interval $I$,

2) at random time $\tau_{h}$, which is managed by the risk level $h\left(X_{t}\right) \geq 0$.

This can be expressed as follows

$$
\left\{\begin{array}{l}
\tau=\tau_{I} \wedge \tau_{h} \\
\tau_{I}=\text { inf } t \geq 0: X_{t} \notin I \\
\tau_{h}=\text { inf } t \geq 0: \int_{0}^{t} h\left(X_{s}\right) d s \geq \varepsilon\left(X, Y_{1}, \ldots, Y_{l}, Z_{1}, \ldots, Z_{n}\right), \varepsilon \sim \exp (1)
\end{array}\right.
$$

Note that the random variable $\varepsilon$ is independent of $\left(X, Y_{1}, \ldots, Y_{l}, Z_{1}, \ldots, Z_{n}\right)$.

To track $\tau_{h}$, we use the process indicator $D_{t}=\mathbb{I}_{t \geq \tau_{h}}$, where $\mathbb{D}=\mathfrak{D}_{t}, t \geq 0$, is a filter generated by $D$ and $\mathbb{F}=\mathfrak{F}_{t}, t \geq 0$ is filter generator $\left(W^{x}, W^{y_{1}}, \ldots W^{y_{l}}, W^{z_{1}}, \ldots, W^{z_{n}}\right)$. We use the filtering $\mathbb{G}=\mathfrak{G}_{t}, t \geq 0$, where $\mathfrak{G}_{t}=\mathfrak{F}_{t} \bigvee \mathfrak{D}_{t}$. Note that $\left(X, Y_{1}, \ldots, Y_{l}, Z_{1}, \ldots, Z_{n}\right)$ are applied to $\mathbb{G}$ and $\tau$ is a stopping time $\left(\tau \leq t \in \mathfrak{G}_{t}\right.$ for all $\left.t \geq 0\right)$.

We will evaluate the derivative asset of some payoff (payment) using the neutral pricing risk and Markovian chain $X$, the price $u^{\bar{\varepsilon}, \bar{\delta}^{\prime}}\left(t, x, y_{1}, \ldots y_{l}, z_{1}, \ldots, z_{r}\right)$ of some derivative assets at the initial moment of time has the form

$$
u^{\bar{\varepsilon}, \bar{\delta}^{\prime}}\left(t, x, y_{1}, \ldots y_{l}, z_{1}, \ldots, z_{r}\right)=\widetilde{\mathbb{E}}_{x, y_{1}, \ldots y_{l}, z_{1}, \ldots, z_{r}}\left[\exp \left(-\int_{0}^{t} r\left(X_{s}\right) d s\right) H\left(X_{t} \mathbb{I}_{t>\tau}\right)\right],
$$

where $\bar{\varepsilon}=\left(\varepsilon_{1}, \ldots, \varepsilon_{l}\right), \overline{\delta^{\prime}}=\left(\delta_{1}, \ldots, \delta_{r}\right)$, and $\left(x, y_{1}, \ldots y_{l}, z_{1}, \ldots, z_{r}\right) \in E$ is a starting point of the process $\left(X, Y_{1}, \ldots, Y_{l}, Z_{1}, \ldots, Z_{r}\right)$. Using the Feynmann-Kac formulas, we can show that $u^{\bar{\varepsilon}, \bar{\delta}^{\prime}}\left(t, x, y_{1}, \ldots y_{l}, z_{1}, \ldots, z_{r}\right)$ satisfies the following Cauchy problem (see [9])

$$
\begin{gathered}
\left(-\partial_{t}+\mathfrak{L}^{\bar{\varepsilon}, \overline{\delta^{\prime}}}\right) u^{\bar{\varepsilon}, \overline{\delta^{\prime}}}=0, \quad\left(y_{1}, \ldots y_{l}, z_{1}, \ldots, z_{r}\right) \in E, t \in \mathbb{R}^{+}, \\
u^{\bar{\varepsilon}, \bar{\delta}^{\prime}}\left(0, x, y_{1}, \ldots y_{l}, z_{1}, \ldots, z_{r}\right)=H(x),
\end{gathered}
$$

where the operator $\mathfrak{L}^{\bar{\varepsilon}, \overline{\delta^{\prime}}}$ has the form

$$
\begin{gathered}
\mathfrak{L}^{\bar{\varepsilon}, \bar{\delta}^{\prime}}=\sum_{j=1}^{l} \frac{1}{\varepsilon_{j}} \mathfrak{L}_{0 j}+\sum_{j=1}^{l} \frac{1}{\sqrt{\varepsilon_{j}}} \mathfrak{L}_{1 j}+\mathfrak{L}_{2 j}+\sum_{i, j} \sqrt{\frac{\delta_{i}}{\varepsilon_{j}}} \mathfrak{M}_{3 i j}+\sum_{i} \sqrt{\delta_{i}} \mathfrak{M}_{1 i}+\sum_{i} \delta_{i} \mathfrak{M}_{2 i}, \\
\mathfrak{L}_{0 j}=\frac{1}{2} \beta_{j}^{2}\left(y_{j}\right) \partial_{y_{j} y_{j}}^{2}+\alpha_{j}\left(y_{j}\right) \partial_{y_{j}, \quad j=\overline{1, l}} \\
\mathfrak{L}_{1 j}=\beta_{j}\left(y_{j}\right)\left(\rho_{x y_{j}} a(x) f\left(y_{1}, \ldots y_{l}, z_{1}, \ldots, z_{r}\right) \partial_{x}-\Lambda_{j}\left(y_{1}, \ldots y_{l}, z_{1}, \ldots, z_{r}\right)\right) \partial_{y_{j}{ }^{\prime}} \\
+\left(b(x)-a(x) \Omega\left(y_{1}, \ldots y_{l}, z_{1}, \ldots, z_{r}\right) f\left(y_{1}, \ldots y_{l}, z_{1}, \ldots, z_{r}\right)\right) \partial_{x}-k(x), \\
\mathfrak{L}_{2 j}=\frac{1}{2} a^{2}(x) f^{2}\left(y_{1}, \ldots y_{l}, z_{1}, \ldots, z_{r}\right) \partial_{x x}^{2} \\
\mathfrak{M}_{1 i}=g_{i}\left(z_{i}\right)\left(\rho_{x z_{i}} a(x) f\left(y_{1}, \ldots y_{l}, z_{1}, \ldots, z_{r}\right) \partial_{x}-\Gamma_{i}\left(y_{1}, \ldots y_{l}, z_{1}, \ldots, z_{r}\right)\right) \partial_{z_{i}}, \\
\mathfrak{M}_{2 i}=\frac{1}{2} g_{i}^{2}\left(z_{i}\right) \partial_{z_{j} z_{i}}^{2}+c_{i}\left(z_{i}\right) \partial_{z_{i}}, k(x)=r(x)+h(x), \quad \mathfrak{L}_{0 j}=\mathfrak{L}_{Y_{j}}^{1} .
\end{gathered}
$$


We assume that the diffusion with the infinitesimal generator $\mathfrak{L}_{Y_{j}}^{1}$ has an invariant distribution $\Pi$ with density

$$
\pi_{j}\left(y_{j}\right)=\frac{2}{\beta_{j}^{2}\left(y_{j}\right)} \exp \int_{y_{j 0}}^{y_{j}} \frac{2 \alpha_{j}(\theta)}{\beta_{j}^{2}(\theta)} d \theta, \quad \forall j=\overline{1, l} .
$$

Besides the initial condition (3), the function $u^{\bar{\varepsilon}, \delta^{\prime}}\left(t, x, y_{1}, \ldots y_{l}, z_{1}, \ldots, z_{r}\right)$ must meet boundary conditions at the points $e_{1}$ and $e_{2}$ of the interval $I$. The boundary conditions at points $e_{1}$ and $e_{2}$ belong to the domain $\mathfrak{L}^{\bar{\varepsilon}, \bar{\delta}^{\prime}}$ and will depend on the nature of process $X$ on the points of $I$ and are classified as natural, output, input or regular [10]. The Cauchy problem (2)-(3) for $\left(f, \alpha_{1}, \ldots, \alpha_{l}, \beta_{1}, \ldots, \beta_{r}, \Lambda_{1}, \ldots, \Lambda_{l}, c_{1}, \ldots, c_{r}, g_{1}, \ldots, g_{r}, \Gamma_{1}, \ldots, \Gamma_{r}\right)$ has no analytical solution. However, for fixed $\overline{\delta^{\prime}}$, the conditions containing $\bar{\varepsilon}$ and are arbitrarily deviated in the $\bar{\varepsilon}$-axis, which causes singular perturbations. For a fixed $\varepsilon_{j}$ condition containing $\delta_{i}$ are small for some small $\overline{\delta^{\prime}}$-axis, which causes regular perturbations. Thus, the $\bar{\varepsilon}$-axis and $\overline{\delta^{\prime}}$-axis yields the combined singular-regular perturbation of $\mathcal{O}(1)$ of the operator $\mathfrak{L}_{2}$. To find the asymptotic solution of the Cauchy problem (2)-(3), we develop $u^{\bar{\varepsilon}, \bar{\delta}^{\prime}}$ in orders $\sqrt{\varepsilon_{j}}$ and $\sqrt{\delta_{i}}$ [11]:

$$
u^{\bar{\varepsilon}, \bar{\delta}^{\prime}}=\sum_{i_{1} \geq 0} \cdots \sum_{i_{l} \geq 0} \sum_{j_{1} \geq 0} \cdots \sum_{j_{r} \geq 0}{\sqrt{\varepsilon_{1}}}^{j_{1}} \ldots{\sqrt{\varepsilon_{l}}}^{j_{l}}{\sqrt{\delta_{1}}}^{i_{1}} \ldots{\sqrt{\delta_{r}}}^{i_{r}} u_{j_{1}, \ldots, j_{n}, i_{1}, \ldots, i_{l}}
$$

where

$$
\begin{aligned}
& \sum_{i_{1} \geq 0} \ldots \sum_{i_{l} \geq 0} \sum_{j_{1} \geq 0} \cdots \sum_{j_{r} \geq 0}{\sqrt{\varepsilon_{1}}}^{j_{1}} \ldots{\sqrt{\varepsilon_{l}}}^{j_{l}}{\sqrt{\delta_{1}}}^{i_{1}} \ldots{\sqrt{\delta_{r}}}^{i_{r}} u_{j_{1}, \ldots, j_{r}, i_{1}, \ldots, i_{l}} \\
& =\lim \sum_{i_{1} \geq 0}^{m_{1}} \cdots \sum_{i_{l} \geq 0}^{m_{l} \geq 0} \sum_{j_{1} \geq 0}^{m_{l+1} \geq 0} \cdots \sum_{j_{n} \geq 0}^{m_{l+r}} \sqrt{\varepsilon_{1}} j_{1} \ldots{\sqrt{\varepsilon_{l}}}^{j_{l}}{\sqrt{\delta_{1}}}^{i_{1}} \ldots{\sqrt{\delta_{r}}}^{i_{r}} u_{j_{1}, \ldots, j_{r}, i_{1}, \ldots, i_{l}}, \quad \forall m_{n} \rightarrow \infty .
\end{aligned}
$$

The approximate price is calculated

$$
u^{\bar{\varepsilon}, \bar{\delta}^{\prime}} \approx u_{\overline{0}, \overline{0^{\prime}}}+\sum_{j=1}^{l} \sqrt{\varepsilon_{j}} u_{\overline{j_{j}, 0^{\prime}}}+\sum_{i=1}^{r} \sqrt{\delta_{i}} u_{\overline{0}, \overline{1_{i}^{\prime}}} .
$$

The choice of development in half-integer orders $\varepsilon_{j}$ and $\delta_{i}$ are natural for $\mathfrak{L}^{\bar{\varepsilon},}, \delta^{\prime}$.

By conducting an analysis of singular perturbations at the corresponding levels, we obtain that $u_{\overline{0}, \overline{0}^{\prime}}, u_{\overline{1}, \overline{0}^{\prime}}, u_{\overline{0}, \overline{1_{i}^{\prime}}}$ do not depend on $y_{1}, \ldots, y_{l}$. The basic findings of the asymptotic analysis are given using the following formulas

$$
\begin{gathered}
\mathcal{O}(1): \sum_{j=1} \mathfrak{L}_{0 j} u_{\overline{2}_{j}, \overline{0}^{0^{\prime}}}+\left(-\partial_{t}+\left\langle\mathfrak{L}_{2}\right\rangle\right) u_{\overline{0}, \overline{0^{\prime}}}=0, u_{\overline{0}, \overline{0^{\prime}}}\left(0, x, z_{1}, \ldots, z_{r}\right)=H(x), \\
\mathcal{O}\left(\sqrt{\varepsilon_{j}}\right): \mathfrak{L}_{0 j} u_{\overline{3}_{j}, \overline{0^{\prime}}}+\mathfrak{L}_{1 j} u_{\overline{2}_{j}, \overline{0^{\prime}}}+\left(-\partial_{t}+\left\langle\mathfrak{L}_{2}\right\rangle\right) u_{\overline{1}_{j,}, \overline{0^{\prime}}}+\sum_{k \neq j} \mathfrak{L}_{1 k} u_{\overline{1}} \overline{1_{k j}, \overline{0^{\prime}}}+\sum_{i \neq j} \mathfrak{L}_{1 i}=\mathcal{A}_{j} u_{\overline{0}, \overline{0^{\prime}}}, \\
u_{\overline{1}_{j}, \overline{0}^{\prime}}\left(0, x, z_{1}, \ldots, z_{n}\right)=0, \overline{1}_{k j}=(\underbrace{0, \ldots 1}_{k} \underbrace{0,1,0, \ldots 0}_{j}) .
\end{gathered}
$$

According to the analysis of regular perturbations we have

$$
\mathcal{O}\left(\sqrt{\delta_{i}}\right):\left(-\partial_{t}+\left\langle\mathfrak{L}_{2}\right\rangle\right) u_{\overline{0,1_{i}^{\prime}}}=\mathcal{B}_{i} \partial_{z_{i}} u_{\overline{0}, \overline{0^{\prime}}}, u_{\overline{0}, \overline{1_{i}^{\prime}}}\left(0, x, z_{1}, \ldots, z_{r}\right)=0, \quad i=\overline{1, r} .
$$


Operators $\left\langle\mathfrak{L}_{2}\right\rangle, \mathcal{A}_{j}, \mathcal{B}_{i}$ and $\partial_{z_{i}}$ are defined by the formulas

$$
\begin{gathered}
\left\langle\mathfrak{L}_{2}\right\rangle=\frac{1}{2} \bar{\sigma}^{2} a^{2}(x) \partial_{x x}^{2}+(b(x)-\overline{f \Omega} a(x)) \partial_{x}-k(x), \quad x \in\left(e_{1}, e_{2}\right), \\
\mathcal{A}_{j}=-v_{3 j} a(x) \partial_{x} a^{2}(x) \partial_{x x}^{2}-v_{2 j} a^{2}(x) \partial_{x x}^{2}-\mathcal{U}_{2 j} a(x) \partial_{x} a(x) \partial_{x}-\mathcal{U}_{1 j} a(x) \partial_{x}, \\
\mathcal{B}_{i}=-v_{1 i} a(x) \partial_{x}-v_{0 i}, \quad \partial_{z_{i}}=\partial_{z_{i}} \bar{\sigma} \partial_{\bar{\sigma}}+\bar{f}^{\prime} \partial_{\overline{f \Omega}}, \quad v_{1 i}:=g_{i} \rho_{x z_{i}}\langle f\rangle, \quad v_{0}=g_{i}\left\langle\Gamma_{i}\right\rangle, \quad \forall i=\overline{1, n},
\end{gathered}
$$
and norm function is defined by

$$
\begin{gathered}
\langle\mathcal{X}\rangle_{j}:=\int \mathcal{X}\left(y_{1}, \ldots y_{l}\right) \pi_{j}\left(y_{j}\right) d y_{j}, \quad \forall j=\overline{1, l}, \\
\langle\mathcal{X}\rangle_{1,2}=\int_{R^{2}} \mathcal{X}\left(y_{1}, \ldots y_{l}\right) \pi_{1}\left(y_{1}\right) \pi_{2}\left(y_{2}\right) d y_{1} d y_{2}, \ldots, \\
\langle\mathcal{X}\rangle_{l-1, l}=\int_{R^{l}} \mathcal{X}\left(y_{1}, \ldots y_{l}\right) \pi_{1}\left(y_{1}\right) \ldots \pi_{l}\left(y_{l}\right) d y_{1} \ldots d y_{l}, \\
\langle\mathcal{X}\rangle_{l-1, l}=\langle\mathcal{X}\rangle,\langle f \Omega\rangle:=\overline{f \Omega},\left\langle f^{2}\right\rangle=\bar{\sigma}^{2} .
\end{gathered}
$$

We find solutions of the equations (4)-(6) on the basis of eigenfunctions, eigenvalues of the operator $\left\langle\mathfrak{L}_{2}\right\rangle$, each of which meets the corresponding Poisson equation

$$
\begin{gathered}
\mathfrak{L}_{01} \varphi_{1}=f^{2}-\left\langle f^{2}\right\rangle_{1}, \mathfrak{L}_{02} \varphi_{2}=\left\langle f^{2}\right\rangle_{1}-\left\langle f^{2}\right\rangle_{1,2}{ }^{\prime} \ldots, \mathfrak{L}_{0 l} \varphi_{l}=\left\langle f^{2}\right\rangle_{l-2, l-1}-\left\langle f^{2}\right\rangle_{l-1, l^{\prime}} \\
\mathfrak{L}_{01} \eta_{1}=f \Omega-\langle f \Omega\rangle_{1}, \ldots, \mathfrak{L}_{0 j} \eta_{j}=\langle f \Omega\rangle_{j-2, j-1}-\langle f \Omega\rangle_{j-1, j}, \ldots, \mathfrak{L}_{0 l} \eta_{l}=\langle f \Omega\rangle_{l-2, l-1}-\langle f \Omega\rangle_{l-1, l} .
\end{gathered}
$$

Theorem 1. Assume that we can solve the following equation to find an eigenvalue

$$
-\left\langle\mathfrak{L}_{2}\right\rangle \psi_{n}=\lambda_{n} \psi_{n}, \quad \psi_{n} \in \operatorname{dom}\left(\left\langle\mathfrak{L}_{2}\right\rangle\right),
$$

and also that $H \in \mathcal{H}$. Then the solution $u_{\overline{0}, \overline{0^{\prime}}}$ has the form

$$
u_{\overline{0}, \overline{0}^{\prime}}=\sum_{n=1}^{\infty} c_{n} \psi_{n} T_{n}, \quad c_{n}=\left(\psi_{n}, H\right), \quad T_{n}=e^{-t \lambda_{n}} .
$$

Proof. Since $u_{\overline{0}, \overline{0^{\prime}}}$ satisfies the differential equation (4), suppose that occurs (7), the boundary conditions are fulfilled $u_{\overline{0}, \overline{0}^{\prime}}\left(0, x, z_{1}, \ldots, z_{n}\right)=H\left(z_{1}, \ldots, z_{n}\right), T_{n}\left(0, z_{1}, \ldots, z_{n}\right)=1, \varphi(\lambda)=\mathrm{Id}$, this means

$$
\operatorname{Id} f=\sum_{n=1}^{\infty}\left(\psi_{n}, f\right) \psi_{n}, \quad \forall f \in \mathcal{H},
$$

this is equivalent to having its eigenfunctions $\psi_{n}$ dense self-adjoint operators in $\mathcal{H}$ form the Schauder basis. In fact, the basis can be chosen orthonormal $\left(\psi_{n}, \psi_{m}\right)=\delta_{n, m}$. Also note that $\varphi(\lambda)=R_{\lambda}$ gives the actual representation of the resolvent of the operator

$$
R_{\lambda} f=\sum_{n=1}^{\infty} \frac{\left(\psi_{n}, f\right)}{\lambda_{n}-\lambda} \psi_{n}, \quad \forall f \in \mathcal{H}, \lambda \in \rho(\mathfrak{L}),
$$

to payoff function $H$ :

$$
\text { Id } H=\sum_{n=1}^{\infty}\left(\psi_{n}, f\right) \psi_{n}=\sum_{n=1}^{\infty} c_{n} \psi_{n} \text {. }
$$


Theorem 2. Let $c_{n}, \psi_{n}, T_{n}$ be described using Theorem 1. We define

$$
\mathcal{A}_{j k, n}:=\left(\psi_{k}, \mathcal{A}_{j} \psi_{n}\right), \quad U_{k, n}:=\frac{T_{k}-T_{n}}{\lambda_{k}-\lambda_{n}} .
$$

Then the solution $u_{\overline{1}_{j}, \overline{0}^{\prime}}$ of equation (5) has the form

$$
u_{\overline{1}_{j, 0^{\prime}}}=\sum_{n} \sum_{k \neq n} c_{n} A_{j k, n} \psi_{k} U_{k, n}-\sum_{n} c_{n} \mathcal{A}_{j n, n} \psi_{n} t T_{n} .
$$

Note that $u_{\overline{1}_{j}, \overline{0}^{\prime}}$ is linear in the parameter group $\left(\vartheta_{3 j}, \vartheta_{2 j}, u_{2 j}, u_{1 j}\right)$.

Proof. Let us show that $u_{\overline{1}_{j}, \overline{0}^{\prime}}$ satisfies the differential equation and boundary conditions (5). It is clear that the boundary conditions for $u_{\overline{1}_{j, 0^{\prime}}}\left(0, x, z_{1}, \ldots, z_{n}\right)=0$ are executed. To show that $u_{\overline{1}, \overline{0}^{\prime}}$ satisfies the differential equation (6), we note that

$$
\mathcal{A}_{j} u_{\overline{1}_{j}, \overline{0^{\prime}}}=\sum_{n} c_{n}\left(\mathcal{A}_{j} \psi_{n}\right) T_{n}=\sum_{n} \sum_{k} c_{n} \mathcal{A}_{j k, n} \psi_{k} T_{n}
$$

according to the proof of Theorem 1 . Now, using (7) and the following equality

$$
\left(-\partial_{t}-\lambda_{k}\right) U_{k, n}=T_{n}, \quad\left(-\partial_{t}-\lambda_{n}\right) t T_{n}=-T_{n},
$$

it is easy to see that

$$
\left(-\partial_{t}+\left\langle\mathfrak{L}_{2}\right\rangle\right) u_{\overline{1}_{j}, \overline{0^{\prime}}}=\mathcal{A}_{j} u_{\overline{1}_{j}, \overline{0^{\prime}}}=\sum_{n} c_{n}\left(\mathcal{A}_{j} \psi_{n}\right) T_{n}=\sum_{n} \sum_{k} c_{n} \mathcal{A}_{j k, n} \psi_{k} T_{n}
$$

Theorem 3. Let $c_{n}, \psi_{n}$ and $T_{n}$ be defined with Theorem 1, and $U_{k, n}$ with Theorem 2. We have

$$
\widetilde{\mathcal{B}}_{i k, n}:=\left(\psi_{k}, \mathcal{B}_{i} \partial_{Z_{i}} \psi_{n}\right), \mathcal{B}_{i k, n}:=\left(\psi_{k}, \mathcal{B}_{i} \psi_{n}\right), V_{i k, n}:=\frac{T_{k}-T_{n}}{\left(\lambda_{k}-\lambda_{n}\right)^{2}}+\frac{t T_{n}}{\lambda_{k}-\lambda_{n}} .
$$

Then the solution $u_{\overline{0}, \overline{1_{i}^{\prime}}}$ has the form

$$
\begin{aligned}
u_{\overline{0}, \overline{1_{i}^{\prime}}} & =\sum_{n} \sum_{k \neq n} c_{n} \widetilde{\mathcal{B}}_{i k, n} \psi_{k} U_{i k, n}-\sum_{n} c_{n} \widetilde{\mathcal{B}}_{i n, n} \psi_{n} t T_{n} \\
& +\sum_{n} \sum_{k \neq n}\left(\partial_{Z_{i}} c_{n}\right) \mathcal{B}_{i k, n} \psi_{k} U_{i k, n}-\sum_{n}\left(\partial_{Z_{i}} c_{n}\right) \mathcal{B}_{i n, n} \psi_{n} t T_{n} \\
& +\sum_{n} \sum_{k \neq n} c_{n} \mathcal{B}_{i k, n} \psi_{k}\left(\partial_{Z_{i}} \lambda_{n}\right) V_{i k, n}-\sum_{n} c_{n} \mathcal{B}_{i n, n} \psi_{n}\left(\partial_{Z_{i}} \lambda_{n}\right) \frac{1}{2} t^{2} T_{n} .
\end{aligned}
$$

Proof. We need to show that $u_{\overline{0}, \overline{1}_{i}^{\prime}}$ satisfies the differential equation and boundary conditions (5). We see that the boundary condition $u_{\overline{0}, \overline{1_{i}^{\prime}}}\left(0, x, z_{1}, \ldots, z_{n}\right)=0$ is executed. To show that $u_{\overline{0}, \overline{1}_{i}^{\prime}}$ satisfies the differential equation, we note that

$$
\begin{aligned}
\mathcal{B}_{i} \partial_{z_{i}} u_{\overline{0}, \overline{0^{\prime}}} & =\sum_{n} c_{n}\left(\mathcal{B}_{i} \partial_{z_{i}} \psi_{n}\right) T_{n}+\sum_{n}\left(\partial_{z_{i}} c_{n}\right)\left(\mathcal{B}_{i} \psi_{n}\right) T_{n} \\
& +\sum_{n} c_{n}\left(\mathcal{B}_{i} \psi_{n}\right)\left(\partial_{z_{i}} T_{n}\right)=\sum_{n} \sum_{k} c_{n} \widetilde{\mathcal{B}}_{i k, n} \psi_{k} T_{n} \\
& +\sum_{n} \sum_{k}\left(\partial_{z_{i}} c_{n}\right)\left(\mathcal{B}_{i k, n} \psi_{k}\right) T_{n}-\sum_{n} \sum_{k} c_{n} \mathcal{B}_{i k, n} \psi_{k}\left(\partial_{z_{i}} \lambda_{n}\right) t T_{n}, \quad i=\overline{1, n}
\end{aligned}
$$


where we used

$$
\text { Id } H=\sum_{n=1}^{\infty}\left(\psi_{n}, f\right) \psi_{n}=\sum_{n=1}^{\infty} c_{n} \psi_{n},
$$

in the second equality using $-\left\langle\mathfrak{L}_{2}\right\rangle \psi_{n}=\lambda_{n} \psi_{n}$ and equality

$$
\begin{gathered}
\left(-\partial_{t}-\lambda_{k}\right) U_{k, n}=T_{n}, \quad\left(-\partial_{t}-\lambda_{n}\right) t T_{n}=-T_{n}, \\
\left(-\partial_{t}-\lambda_{k}\right) V_{k, n}=-t T_{n}, \quad\left(-\partial_{t}-\lambda_{k}\right) \frac{1}{2} t^{2} T_{n}=-t T_{n},
\end{gathered}
$$

one can see that

$$
\begin{gathered}
\left(-\partial_{t}+\left\langle\mathfrak{L}_{2}\right\rangle\right) u_{\overline{0}, \overline{1_{i}^{\prime}}}=\mathcal{B}_{i} \partial_{z_{i}} u_{\overline{0}, \overline{0^{\prime}}}=\sum_{n} c_{n}\left(\mathcal{B}_{n} \partial_{z_{n}} \psi_{n}\right) T_{n} \\
\sum_{n}\left(\partial_{z_{n}} c_{n}\right)\left(\mathcal{B}_{n} \psi_{n}\right) T_{n}+\sum_{n} c_{n}\left(\mathcal{B}_{n} \psi_{n}\right)\left(\partial_{z_{n}} T_{n}\right)=\sum_{n} \sum_{k} c_{n} \widetilde{\mathcal{B}}_{i k, n} \psi_{k} T_{n} \\
\left.+\sum_{n} \sum_{k} \partial_{z_{i}} c_{n}\right) \mathcal{B}_{i k, n} \psi_{k} T_{n}-\sum_{n} \sum_{k} c_{n} \mathcal{B}_{i k, n} \psi_{k}\left(\partial_{z_{i}} \lambda_{n}\right) t T_{n}, \quad i=\overline{1, n} .
\end{gathered}
$$

Note that $u_{\overline{0}, \overline{1_{i}^{\prime}}}$ is linear in $\left(v_{1 i}{\overline{\sigma^{\prime}}}^{\prime}, v_{1 i} \overline{f \Omega^{\prime}}, v_{0 i} \bar{\sigma}^{\prime}, v_{0 i} \overline{f \Omega^{\prime}}\right)$.

We have obtained the approximate solution $u^{\bar{\varepsilon}, \overline{\delta^{\prime}}} \approx u_{\overline{0}, \overline{0^{\prime}}}+\sum_{j=1}^{l} \sqrt{\varepsilon_{j}} u_{\overline{1_{j}}, \overline{0^{\prime}}}+\sum_{i=1}^{n} \sqrt{\delta_{i}} u_{\overline{,}, \overline{1_{i}^{\prime}}}$ for the derivative asset pricing.

For a more exact result we assume that the Payoff function $H(x)$ and its derivative are smooth and limited functions. Thus, we restrict our derivative analysis to a smooth and limited payoff; in this case, the closeness estimates is based on the following theorem.

Theorem 4. For the fixed $\left(t, x, y_{1}, \ldots, y_{l}, z_{1}, \ldots, z_{r}\right)$ there exists an invariable $C$ such that for any $\varepsilon_{j} \leq 1, \delta_{i} \leq 1$ we have

$$
\left|u^{\bar{\varepsilon}, \overline{\delta^{\prime}}}-\left(u_{\overline{0}, \overline{0^{\prime}}}+\sum_{j=1}^{l} \sqrt{\varepsilon_{j}} u_{\overline{1_{j}}, \overline{0^{\prime}}}+\sum_{i=1}^{n} \sqrt{\delta_{i}} u_{\overline{0}, \overline{1_{i}^{\prime}}}\right)\right| \leq C\left(\sum_{j=1}^{l} \varepsilon_{j}+\sum_{i=1}^{r} \delta_{i}\right) .
$$

Proof. Before setting the main result of accuracy we formulate such a lemma.

Lemma 1. Let $J\left(y_{1}, \ldots, y_{l}, z_{1}, \ldots, z_{n}\right)$ grows polynomially. Then for every $\left(y_{1}, \ldots, y_{l}, z_{1}, \ldots, z_{n}\right)$, $s<t$, there is a positive start $C<\infty$ such that for any $\varepsilon_{j} \leq 1, \delta_{i} \leq 1$, the following inequality holds

$$
\widetilde{\mathbb{E}}_{y_{1}, \ldots, y_{l}, z_{1}, \ldots, z_{n}}\left[\left|J\left(Y_{1 s}, \ldots, Y_{l s}, Z_{1 s}, \ldots, Z_{n s}\right)\right|\right] \leq C .
$$

Proof. It is enough to consider $J\left(y_{1}, \ldots, y_{l}, z_{1}, \ldots, z_{n}\right)=y_{j}^{k}$ and $J\left(y_{1}, \ldots, y_{l}, z_{1}, \ldots, z_{n}\right)=z_{i}^{k}$, $k \in \mathbb{N}$. For the second one we have the following. Physically $\mathbb{P}$ we understand as

$$
\mathbb{E}\left[\left|Z_{i s}\right|^{k}\right]=\mathbb{E}\left[\left|Z_{i \delta_{i} s}^{(1)}\right|^{k}\right] \leq \sup _{\delta_{i} \leq 1} \mathbb{E}\left[\left|Z_{i \delta_{i} s}^{(1)}\right|^{k}\right] \leq C_{i}(s, k) \leq C_{i}(t, k), \quad i=\overline{1, n} .
$$

Now we define exponential martingales $M_{t}^{\left(\Gamma_{\mathrm{i}}\right)}$, which connect the dynamics $Z_{i}$ at neutral risk of measurement $\widetilde{\mathbb{P}}$ in its dynamics according to physical measure $\mathbb{P}$. We have

$$
M_{t}^{\Gamma_{i}}:=\exp \left(-\int_{0}^{t} \Gamma_{i}\left(Y_{1 s}, \ldots, Y_{l s}, Z_{1 s}, \ldots, Z_{n s}\right) d W_{s}^{z_{i}}-\frac{1}{2} \int_{0}^{t} \Gamma_{i}^{2}\left(Y_{1 s}, \ldots, Y_{l s}, Z_{1 s}, \ldots, Z_{n s}\right) d s\right)=\left.\frac{d \widetilde{\mathbb{P}}}{d \mathbb{P}}\right|_{\mathcal{F}_{t}} .
$$


$\left|Z_{S}\right|^{k}$ can be found as follows

$$
\begin{aligned}
\widetilde{\mathbb{E}}\left[\left|Z_{i s}\right|^{k}\right] & =\mathbb{E}\left[\left|Z_{i s}\right|^{k} M_{s}^{\left(\Gamma_{\mathrm{i}}\right)}\right] \\
& =\mathbb{E}\left[\left|Z_{i s}\right|^{k} \exp \left(\frac{1}{2} \int_{0}^{s} \Gamma_{\mathrm{i}}^{2}\left(Y_{1 u}, \ldots, Y_{l u}, Z_{1 u}, \ldots, Z_{n u}\right) d u\right)\left(M_{s}^{\left(2 \Gamma_{\mathrm{i}}\right)}\right)^{1 / 2}\right] \\
& \leq\left(\mathbb{E}\left[\left|Z_{i s}\right|^{2 k} \exp \left(\int_{0}^{s} \Gamma_{\mathrm{i}}^{2}\left(Y_{1 u}, \ldots, Y_{l u}, Z_{1 u}, \ldots, Z_{n u}\right) d u\right)\right]\right)^{1 / 2}
\end{aligned}
$$

$\left(\mathbb{E}\left[M_{S}^{\left(2 \Gamma_{\mathrm{i}}\right)}\right]\right)^{\frac{1}{2}} \quad$ (by Cauchy-Schwartz)

$$
=\left(\mathbb{E}\left[\left|Z_{i s}\right|^{2 k} \exp \left(\int_{0}^{s} \Gamma_{\mathrm{i}}^{2}\left(Y_{1 u}, \ldots, Y_{l u}, Z_{1 u}, \ldots, Z_{n u}\right) d u\right)\right]\right)^{1 / 2}
$$

$\left(M^{\left(2 \Gamma_{\mathrm{i}}\right)}-\right.$ is $\mathbb{P}$-martingale $)$

$$
\left.\leq\left(\mathbb{E}\left[\left|Z_{i \delta_{i} s}^{(1)}\right|^{2 k}\right] \exp \left(s\left\|\Gamma_{\mathrm{i}}\right\|_{\infty}^{2}\right)\right)\right)^{\frac{1}{2}} \leq C .
$$

Consider now the case $J\left(y_{1}, \ldots, y_{l}, z_{1}, \ldots, z_{n}\right)=y_{j}^{k}$. We have

$$
\mathbb{E}\left[\left|Y_{j s}\right|^{k}\right]=\mathbb{E}\left[\left|Y_{j s / \varepsilon_{j}}^{(1)}\right|^{k}\right] \leq \sup _{\varepsilon_{j} \leq 1} \mathbb{E}\left[\left|Y_{j s / \varepsilon_{j}}^{(1)}\right|^{k}\right] \leq C_{j}(k) .
$$

Using the above considerations is easy to show that

$$
\left.\widetilde{\mathbb{E}}\left[\left|Y_{j s}\right|^{k}\right]=\mathbb{E}\left[\left|Y_{j s}\right|^{k} M_{s}^{\left(\Lambda_{\mathrm{j}}\right)}\right] \leq\left(\mathbb{E}\left[\left|Y_{j s / \varepsilon_{j},}^{(1)}\right|^{2 k}\right] \exp \left(s\left\|\Lambda_{\mathrm{j}}\right\|_{\infty}^{2}\right)\right)\right)^{\frac{1}{2}} \leq C_{j} .
$$

The Lemma 1 is proved.

Let us return to the proof of the Theorem 4 . We start with the definition of the remainder term $R^{\bar{\varepsilon}, \bar{\delta}^{\prime}}$

$$
\begin{gathered}
u_{\overline{0}, \overline{0^{\prime}}}+\sum_{j=1}^{l} \sqrt{\varepsilon_{j}} u_{\overline{1_{j}}, \overline{0^{\prime}}}+\sum_{i=1}^{n} \sqrt{\delta_{i}} u_{\overline{0}, \overline{1_{i}^{\prime}}}+\sum_{j=1}^{l} \varepsilon_{j}\left(u_{\overline{2_{j}}, \overline{0^{\prime}}}+\sum_{j=1}^{l} \sqrt{\varepsilon_{j}} u_{\overline{3_{j}}, \overline{0^{\prime}}}\right) \\
u^{\bar{\varepsilon}, \overline{\delta^{\prime}}}+\sum_{k \neq j}^{l} \sqrt{\varepsilon_{k}} \sqrt{\varepsilon_{j}} u_{\overline{1_{k j}}, \overline{0^{\prime}}}+\sum_{j=1}^{l} \sum_{i=1}^{n} \sqrt{\delta_{i}}\left(\sqrt{\varepsilon_{j}} u_{\overline{1_{j} 1_{i}^{\prime}}}+\sum_{j=1}^{l} \varepsilon_{j} u_{\overline{2_{j}} \overline{1_{i}^{\prime}}}\right) \\
+\sum_{k \neq j}^{l} \sum_{j=1}^{l} \sum_{i=1}^{n} \sqrt{\delta_{i}} \sqrt{\varepsilon_{k}} \sqrt{\varepsilon_{j}} u_{\overline{1_{k j},}, \overline{1_{i}^{\prime}}}+R^{\bar{\varepsilon}, \overline{\delta^{\prime}}} .
\end{gathered}
$$

Functions $u_{\overline{0}, \overline{0^{\prime}}}, u_{\overline{1_{j}}, \overline{0^{\prime}}}, u_{\overline{0}, \overline{1_{i}^{\prime}}}$ are the only solutions of equations (4)-(6), respectively. Function $u_{\overline{2_{j}}, \overline{0^{\prime}}}, w_{\overline{2_{j},}, \overline{0^{\prime}}}$ is a solution of the Poisson equation $0=\mathfrak{L}_{0 j} u_{\overline{0}, \overline{0^{\prime}}}, j=\overline{1, l}$. To characterize $u_{\overline{1_{j} \overline{1}_{i}^{\prime}}}, u_{\overline{2_{j}} \overline{1}_{i}^{\prime}}$ continue the singular analysis of perturbations.

$$
0=\mathfrak{L}_{0 j} u_{\overline{3_{j}} \overline{1_{i}^{\prime}}}+\mathfrak{L}_{1 j} u_{\overline{2_{j} 1_{i}^{\prime}}}+\sum_{k \neq j}^{l} \mathfrak{L}_{1 k} u_{\overline{1_{k j},} \overline{1_{i}^{\prime}}}+\left(-\partial_{t}+\mathfrak{L}_{2}\right) u_{\overline{1_{j} 1_{i}^{\prime}}} \mathcal{O}+\mathfrak{M}_{3 i j} u_{\overline{2_{j}}, \overline{0^{\prime}}}+\mathfrak{M}_{1 i} u_{\overline{1_{j}}, \overline{0^{\prime}}} .
$$


Equation (8) is a Poisson equation. In order to determine the solution for (8) $u_{\overline{3_{j}} \overline{1_{i}^{\prime}}}$ in the space $L^{2}(\mathbb{R}, \pi)$, the centering condition must meet $0=\mathfrak{L}_{0 j} u+\mathcal{X}, j=\overline{1, l}$. In (8) the condition of centering is

$$
0=\left\langle\mathfrak{L}_{1 j} u_{\overline{\bar{j}_{j} \overline{1}_{i}^{\prime}}}\right\rangle+\left(-\partial_{t}+\left\langle\mathfrak{L}_{2}\right\rangle\right) u_{\overline{\bar{j}_{j} \overline{1}_{i}^{\prime}}}+\left\langle\mathfrak{M}_{3 i j} u_{\overline{2_{j}}, \overline{0^{\prime}}}\right\rangle+\left\langle\mathfrak{M}_{1 i}\right\rangle u_{\overline{j_{j}, 0^{\prime}}}
$$

let us express $u_{\overline{2_{j}} \overline{1_{i}^{\prime}}}$

$$
\begin{gathered}
0=\left(-\partial_{t}+\left\langle\mathfrak{L}_{2}\right\rangle\right) u_{\overline{0}, \overline{1_{i}^{\prime}}}+\left\langle\mathfrak{M}_{1 i}\right\rangle u_{\overline{0}, \overline{0^{\prime}}}-\sum_{j=1}^{l} \mathfrak{L}_{0 j} u_{\overline{2_{j}} \overline{1_{i}^{\prime}}}+\left(-\partial_{t}+\mathfrak{L}_{2}\right) u_{\overline{0}, \overline{1_{i}^{\prime}}} \\
+\mathfrak{M}_{1 i} u_{\overline{0}, \overline{0^{\prime}}}+\sum_{j=1}^{l} \mathfrak{M}_{3 i j} u_{\overline{1_{j}, 0^{\prime}}}+\mathfrak{L}_{1 j} u_{\overline{1_{j} \overline{1}_{i}^{\prime}}}
\end{gathered}
$$

Let us put down similar terms

$$
\begin{aligned}
& 0=\sum_{j=1}^{l} \mathfrak{L}_{0 j} u_{\overline{2_{j}} \overline{1_{i}^{\prime}}}+\left(\mathfrak{L}_{2}-\left\langle\mathfrak{L}_{2}\right\rangle\right) u_{\overline{0}, \overline{1_{i}^{\prime}}}+\mathfrak{M}_{1 i} u_{\overline{0}, \overline{0^{\prime}}}-\left\langle\mathfrak{M}_{1 i}\right\rangle u_{\overline{0}, \overline{0^{\prime}}}=\sum_{j=1}^{l} \mathfrak{L}_{0 j} u_{\overline{2_{j} \overline{1}_{i}^{\prime}}} \\
& +\left(\left(-\frac{1}{2} a^{2}\left(\bar{\sigma}^{2}-f^{2}\right)\right) \partial_{x x}^{2}+a(f \Omega-\overline{f \Omega}) \partial_{x}\right) u_{\overline{0}, \overline{1_{i}^{\prime}}} \\
& +\left[g_{i}\left(\rho_{x z_{i}} a f \partial_{x}-\Gamma_{i}\right) \partial_{z_{i}}-g_{i}\left(\rho_{x z_{i}} a\langle f\rangle \partial_{x}-\left\langle\Gamma_{i}\right\rangle\right) \partial_{z_{i}}\right] u_{\overline{0}, \overline{0^{\prime}}} \\
& =\sum_{j=1}^{l} \mathfrak{L}_{0 j} u_{\overline{2_{j} \overline{1}_{i}^{\prime}}}+\left(\left(-\frac{1}{2} a^{2}\left(\bar{\sigma}^{2}-f^{2} \mp \bar{\sigma}_{1}^{2} \mp \bar{\sigma}_{12}^{2} \mp \ldots \mp \bar{\sigma}_{l-2, l-1}^{2}\right)\right) \partial_{x x}^{2}\right. \\
& \left.+a\left(f \Omega-\overline{f \Omega} \mp \overline{f \Omega}_{1} \mp \overline{f \Omega}_{12} \mp \cdots \mp \overline{f \Omega}_{l-2, l-1}\right) \partial_{x}\right) u_{\overline{0}, \overline{1}_{i}^{\prime}} \\
& +\left[g _ { i } \left(\rho_{x z_{i}} a\left(f-\langle f\rangle \mp\langle f\rangle_{1} \mp\langle f\rangle_{12} \mp \cdots \mp\langle f\rangle_{l-2, l-1}\right) \partial_{x}\right.\right. \\
& \left.\left.-\left(\Gamma-\langle\Gamma\rangle \mp\langle\Gamma\rangle_{1} \mp\langle\Gamma\rangle_{12} \mp \cdots \mp\langle\Gamma\rangle_{l-2, l-1}\right) \partial_{z_{i}}\right) u_{\overline{0}, \overline{0^{\prime}}}\right] \text {. }
\end{aligned}
$$

Consider such systems of Poisson equations

$$
\begin{gathered}
\mathfrak{L}_{01} \varphi_{1}=f^{2}-\bar{\sigma}_{1}^{2}, \quad \mathfrak{L}_{02} \varphi_{2}=\bar{\sigma}_{1}^{2}-\bar{\sigma}_{12}^{2}, \ldots, \quad \mathfrak{L}_{0 j} \varphi_{j}=\bar{\sigma}_{j-2, j-1}^{2}-\bar{\sigma}_{j-1, j}^{2}, \ldots, \\
\mathfrak{L}_{0 l} \varphi_{l}=\bar{\sigma}_{l-2, l-1}^{2}-\bar{\sigma}_{l-1, l}^{2} \mathfrak{L}_{01} \eta_{1}=f \Omega-\bar{f}_{1}, \ldots, \mathfrak{L}_{0 l} \eta_{l}=\overline{f \Omega}_{l-2, l-1}-\overline{f \Omega}, \\
\mathfrak{L}_{01} \xi_{1}=f-\langle f\rangle_{1}, \quad \mathfrak{L}_{02} \xi_{2}=\langle f\rangle_{1}-\langle f\rangle_{12}, \ldots, \mathfrak{L}_{0 l} \xi_{l}=\langle f\rangle_{l-2, l-1}-\langle f\rangle \\
\mathfrak{L}_{01} \zeta_{1}=\Gamma-\langle\Gamma\rangle_{1}, \ldots, \mathfrak{L}_{0 l} \zeta_{l}=\langle\Gamma\rangle_{l-2, l-1}-\langle\Gamma\rangle .
\end{gathered}
$$

Functions $\xi_{j}\left(y_{1}, \ldots, y_{l}, z_{1}, \ldots, z_{n}\right), \eta_{j}\left(y_{1}, \ldots, y_{l}, z_{1}, \ldots, z_{n}\right)$ are solutions of the corresponding Poisson equations, the formula holds

$$
\sum_{j=1}^{l} \mathfrak{L}_{0 j} u_{\overline{2_{j} \overline{1}_{i}^{\prime}}}+\sum_{j=1}^{l} \mathfrak{L}_{0 j}\left(-\frac{1}{2} a^{2} \varphi_{j} \partial_{x x}^{2}+a \eta_{j} \partial_{x}\right) u_{\overline{0,1_{i}^{\prime}}}+\left[g_{i}\left(\rho_{x z_{i}} a \xi_{j} \partial_{x}-\zeta_{j}\right) \partial_{z_{i}}\right] u_{\overline{0}, \overline{0^{\prime}}}=0
$$

Therefore, the formula holds

$$
u_{\overline{2_{j} 1_{i}^{\prime}}}=-\left(\frac{1}{2} a^{2} \varphi_{j} \partial_{x x}^{2}-a \eta_{j} \partial_{x}\right) u_{\overline{0}, \overline{1_{i}^{\prime}}}-g_{j}\left(\rho_{x z_{i}} a \xi_{j} \partial_{x}-\zeta_{j}\right) \partial_{z_{i}} u_{\overline{0}, \overline{0^{\prime}}}+D_{j}, \forall j=\overline{1, l}, \quad i=\overline{1, n} .
$$


$D_{j}\left(x, z_{1}, \ldots, z_{n}\right)$ is a constant that does not depend on $y$. Substituting (10) in (9) we find $u_{\overline{1_{j}}} \overline{1_{i}^{\prime}}$ knowing $u_{\overline{0}, \overline{0^{\prime}}}, u_{\overline{1_{j}}, \overline{0^{\prime}}}, u_{\overline{0}, \overline{1_{i}^{\prime}}} \cdot u_{\overline{1_{j}} \overline{1_{i}^{\prime}}}$ is a solution (9) with boundary conditions $u\left(0, x, z_{1}, \ldots, z_{n}\right)=0$.

Take $u \overline{\overline{1 k}_{j} 0^{\prime}} \equiv 0, \forall k \neq j, k=\overline{1, l}$.

Let us calculate

$$
\begin{aligned}
0 & =\left(-\partial_{t}+\mathfrak{L}^{\bar{\varepsilon}, \overline{\delta^{\prime}}}\right) u^{\bar{\varepsilon}, \overline{\delta^{\prime}}}\left(-\partial_{t}+\mathfrak{L}^{\bar{\varepsilon}, \overline{\delta^{\prime}}}\right) R^{\bar{\varepsilon}, \bar{\delta}^{\prime}}+\sum_{j=1}^{l} \frac{1}{\varepsilon_{j}} F_{0 j}+\sum_{j=1}^{l} \frac{1}{\sqrt{\varepsilon_{j}}} F_{1 j}+\sum_{j=1}^{l} F_{2 j} \\
& +\sum_{i=1}^{n} \sum_{j=1}^{l} \sqrt{\delta_{i}}\left(\frac{1}{\varepsilon_{j}} F_{3 j i}+\frac{1}{\sqrt{\varepsilon_{j}}} F_{4 j i}+F_{5 j i}\right)+\sum_{j=1}^{l} \varepsilon_{j} R_{1 j}^{\varepsilon_{j}}+\sum_{i=1}^{n} \sum_{j=1}^{l} \sqrt{\varepsilon_{j} \delta_{i}} R_{2 j}^{\varepsilon_{j}}+\sum_{i=1}^{n} \delta_{i} R_{3 j}^{\varepsilon_{j}},
\end{aligned}
$$

where

$$
\begin{aligned}
& F_{0 j}=\mathfrak{L}_{0 j} u_{\overline{0}, \overline{0^{\prime}}}, \quad F_{1 j}=\mathfrak{L}_{0 j} u_{\overline{1_{j}}, \overline{0}^{\prime}}+\mathfrak{L}_{1 j} u_{\overline{0}, \overline{0^{\prime}}} \\
& \sum_{j=1}^{l} F_{2 j}=\sum_{j=1}^{l} \mathfrak{L}_{0 j} u_{\overline{2_{j}, 0^{\prime}}}+\sum_{j=1}^{l} \mathfrak{L}_{1 j} u_{\overline{1_{j}, 0^{\prime}}}+\left(-\partial_{t}+\mathfrak{L}_{2}\right) u_{\overline{0,0^{\prime}}} \\
& F_{3 j i}=\mathfrak{L}_{0 j} u_{\overline{0}, \overline{1_{i}^{\prime}}} \\
& F_{4 j i}=\mathfrak{L}_{0 j} u_{\overline{1_{j} \overline{1}_{i}^{\prime}}}+\mathfrak{L}_{1 j} u_{\overline{0}, \overline{1_{i}^{\prime}}}+\mathfrak{M}_{3 i j} u_{\overline{0}, \overline{0^{\prime}}} \\
& \sum_{j=1}^{l} F_{5 j i}=\sum_{j=1}^{l} \mathfrak{L}_{0 j} u_{\overline{2_{j} \overline{1}_{i}^{\prime}}}+\sum_{k \neq j} \mathfrak{L}_{1 j} u_{\overline{1_{j} \overline{1}_{i}^{\prime}}}+\mathfrak{M}_{3 i j} u_{\overline{1_{j}, 0^{\prime}}}+\mathfrak{M}_{1 i} u_{\overline{0}, \overline{0^{\prime}}}+\left(-\partial_{t}+\mathfrak{L}_{2}\right) u_{\overline{0}, \overline{1_{i}^{\prime}}}, \\
& R_{1 j}^{\varepsilon_{j}}=\left(-\partial_{t}+\mathfrak{L}_{2}\right) u_{\overline{2_{j},}, \overline{0^{\prime}}}+\mathfrak{L}_{1 j} u_{\overline{\overline{3}_{j}, 0^{\prime}}}+\sqrt{\varepsilon_{j}}\left(-\partial_{t}+\mathfrak{L}_{2}\right) u_{\overline{3_{j}, 0^{\prime}}}, \\
& R_{2 i j}^{\varepsilon_{j}}=\left(-\partial_{t}+\mathfrak{L}_{2}\right) u_{\overline{1_{j}} \overline{1_{i}^{\prime}}}+\mathfrak{L}_{1 j} u_{\overline{2_{j}} \overline{1_{i}^{\prime}}}+\mathfrak{M}_{1 i} u_{\overline{1_{j}}, \overline{0^{\prime}}}+\mathfrak{M}_{3 i j} u_{\overline{2_{j}}, \overline{0^{\prime}}} \\
& +\sqrt{\varepsilon_{j}}\left(\left(-\partial_{t}+\mathfrak{L}_{2}\right) u_{\overline{j_{j}}, \overline{1^{\prime}}}+\mathfrak{M}_{1 i} u_{\overline{2_{j}}, \overline{0^{\prime}}}+\mathfrak{M}_{3 j} u_{\overline{3_{i j}}, \overline{0^{\prime}}}\right)+\varepsilon_{j} \mathfrak{M}_{1 j} u_{\overline{3_{j}}, \overline{0^{\prime}}}, \\
& R_{3 i j}^{\varepsilon_{j}}=\mathfrak{M}_{1 j} u_{\overline{0}, \overline{1_{i}^{\prime}}}+\mathfrak{M}_{2 j} u_{\overline{0}, \overline{0^{\prime}}}+\mathfrak{M}_{3 j} u_{\overline{1_{j}} \overline{1_{i}^{\prime}}} \\
& +\sqrt{\varepsilon_{j}}\left(\mathfrak{M}_{1 j} u_{\overline{1_{j} 1_{i}^{\prime}}}+\mathfrak{M}_{2 j} u_{\overline{1_{j}}, \overline{0^{\prime}}}+\mathfrak{M}_{3 j} u_{\overline{1_{j} \overline{1}_{i}^{\prime}}}\right)+\varepsilon_{j}\left(\mathfrak{M}_{1 j} u_{\overline{2_{j}} \overline{1_{i}^{\prime}}}+\mathfrak{M}_{2 j} u_{\overline{2_{j}}, \overline{0^{\prime}}}\right) \text {. }
\end{aligned}
$$

It is easy to see, $F_{0 j}=F_{1 j}=F_{3 i j}=F_{4 i j}=F_{5 i j}=0$.

So we have

$$
\begin{gathered}
0=\left(-\partial_{t}+\mathfrak{L}^{\bar{\varepsilon}, \overline{\delta^{\prime}}}\right) R^{\bar{\varepsilon}, \overline{\delta^{\prime}}}+\sum_{j=1}^{l} \varepsilon_{j} R_{1 j}^{\varepsilon_{j}}+\sum_{j=1}^{l} \sum_{i=1}^{n}\left(\sqrt{\varepsilon_{j} \delta_{i}} R_{2 i j}^{\varepsilon_{j}}+\delta_{i} R_{3 i j}^{\varepsilon_{j}}\right) \\
R^{\bar{\varepsilon}, \bar{\delta}^{\prime}}\left(0, x, y_{1}, \ldots, y_{l}, z_{1}, \ldots, z_{n}\right)=\sum_{j=1}^{l} \varepsilon_{j} G_{1 j}^{\varepsilon_{j}}\left(x, y_{1}, \ldots, y_{l}, z_{1}, \ldots, z_{n}\right) \\
+\sum_{j=1}^{l} \sum_{i=1}^{n} \sqrt{\varepsilon_{j} \delta_{i}} G_{2 i j}^{\varepsilon_{j}}\left(x, y_{1}, \ldots, y_{l}, z_{1}, \ldots, z_{n}\right)
\end{gathered}
$$

where

$$
\begin{aligned}
G_{1 j}^{\varepsilon_{j}}\left(x, y_{1}, \ldots, y_{l}, z_{1}, \ldots, z_{n}\right):= & -u_{\overline{2_{j}}, \overline{0^{\prime}}}\left(0, x, y_{1}, \ldots, y_{l}, z_{1}, \ldots, z_{n}\right) \\
& -\sqrt{\varepsilon_{j}} u_{\overline{3_{j}, 0^{\prime}}}\left(0, x, y_{1}, \ldots, y_{l}, z_{1}, \ldots, z_{n}\right),
\end{aligned}
$$




$$
\begin{aligned}
G_{2 i j}^{\varepsilon_{j}}\left(x, y_{1}, \ldots, y_{l}, z_{1}, \ldots, z_{n}\right):= & -u_{\overline{1_{j}} \overline{1_{i}^{\prime}}}\left(0, x, y_{1}, \ldots, y_{l}, z_{1}, \ldots, z_{n}\right) \\
& -\sqrt{\varepsilon_{j}} u_{\overline{2_{j}} \overline{1_{i}^{\prime}}}\left(0, x, y_{1}, \ldots, y_{l}, z_{1}, \ldots, z_{n}\right) .
\end{aligned}
$$

Using the formulas of Feynman-Kats, let us express $R^{\bar{\varepsilon}, \bar{\delta}^{\prime}}\left(t, x, y_{1}, \ldots, y_{l}, z_{1}, \ldots, z_{n}\right)$ solving equation (11) with boundary conditions as a mathematical expectation

$$
\begin{gathered}
R^{\bar{\varepsilon}, \bar{\delta}^{\prime}}\left(t, x, y_{1}, \ldots, y_{l}, z_{1}, \ldots, z_{n}\right) \\
=\sum_{j=1}^{l} \varepsilon_{j} \widetilde{\mathbb{E}}_{x, y_{1}, \ldots, y_{l}, z_{1}, \ldots, z_{n}}\left[e^{-\int_{0}^{t} k\left(X_{s}\right) d s} G_{1 j}^{\varepsilon_{j}}\left(X_{t}, Y_{1 t}, \ldots, Y_{l t}, Z_{1 t}, \ldots, Z_{n t}\right)\right] \\
+\int_{0}^{t} e^{-\int_{0}^{s} k\left(X_{u}\right) d u} R_{1 j}^{\varepsilon_{j}}\left(s, X_{s}, Y_{1 s}, \ldots, Y_{l s}, Z_{1 s}, \ldots, Z_{n s}\right) d s+\sqrt{\varepsilon \delta} \widetilde{\mathbb{E}}_{x, y_{1}, \ldots, y_{l}, z_{1}, \ldots, z_{n}} \\
{\left[e^{-\int_{0}^{t} k\left(X_{s}\right) d s} G_{2}^{\varepsilon}\left(X_{t}, Y_{1 t}, \ldots, Y_{l t}, Z_{1 t}, \ldots, Z_{n t}\right)\right.} \\
\left.+\int_{0}^{t} e^{-\int_{0}^{s} k\left(X_{u}\right) d u} R_{2 i j}^{\varepsilon_{j}}\left(s, X_{s}, Y_{1 s}, \ldots, Y_{l s}, Z_{1 s}, \ldots, Z_{n s}\right) d s\right] \\
+\delta \widetilde{\mathbb{E}}_{x, y_{1}, \ldots, y_{l}, z_{1}, \ldots, z_{n}}\left[e^{-\int_{0}^{s} k\left(X_{u}\right) d u} R_{3 i j}^{\varepsilon_{j}}\left(s, X_{s}, Y_{1 s}, \ldots, Y_{l s}, Z_{1 s}, \ldots, Z_{n s}\right) d s\right] .
\end{gathered}
$$

We can conclude that the functions $\left(R_{1 j}^{\varepsilon_{j}}, R_{2 i j}^{\varepsilon_{j}}, R_{3 i j}^{\varepsilon_{j}}, G_{1 j}^{\varepsilon_{j}}, G_{2 i j}^{\varepsilon_{j}}\right)$ limited by $x$ and polynomially increase by $\left(y_{1}, \ldots, y_{l}, z_{1}, \ldots, z_{n}\right)$ [4]. Thus, according to Lemma 1 we have

$$
\left|R^{\bar{\varepsilon}, \overline{\delta^{\prime}}}\right| \leq \sum_{j=1}^{l} \varepsilon_{j} C_{1 j}+\sum_{j=1}^{l} \sum_{i=1}^{n} \sqrt{\varepsilon_{j} \delta_{i}} C_{2 i j}+\sum_{i=1}^{n} \delta_{i} C_{3 i} \leq\left(\sum_{j=1}^{l} \varepsilon_{j}+\sum_{i=1}^{n} \delta_{i}\right) C_{4} .
$$

and hence

$$
\begin{aligned}
& \left|u^{\bar{\varepsilon}, \overline{\delta^{\prime}}}-\left(u_{\overline{0}, \overline{0^{\prime}}}+\sum_{j=1}^{l} \varepsilon_{j} u_{\overline{j_{j}}, \overline{0^{\prime}}}+\sum_{i=1}^{n} \delta_{i} u_{\overline{0}, \overline{1_{i}^{\prime}}}\right)\right| \leq\left|R^{\bar{\varepsilon}, \overline{\delta^{\prime}}}\right| \\
& +\left|\sum_{j=1}^{l} \varepsilon_{j} u_{\overline{z_{j}}, \overline{0^{\prime}}}+\sum_{j=1}^{l} \varepsilon_{j}^{3 / 2} u_{\overline{j_{j}}, \overline{0^{\prime}}}+\sum_{j=1}^{l} \sum_{i=1}^{n} \sqrt{\varepsilon_{j} \delta_{i}} u_{\overline{1_{j}} \overline{1_{i}^{\prime}}}+\sum_{j=1}^{l} \sum_{i=1}^{n} \sqrt{\delta_{i} \varepsilon} u_{j} \overline{2_{j} \overline{1}_{i}^{\prime}}\right| \\
& \leq\left(\sum_{j=1}^{l} \varepsilon_{j}+\sum_{i=1}^{n} \delta_{i}\right) C_{4}+\sum_{j=1}^{l} \varepsilon_{j}\left|u_{\overline{2_{j}}, \overline{0^{\prime}}}+\sqrt{\varepsilon_{j}} u_{\overline{3_{j}}, \overline{0^{\prime}}}\right| \\
& +\sum_{j=1}^{l} \sum_{i=1}^{n} \sqrt{\varepsilon_{j} \delta_{i}}\left|u_{\overline{1_{j}} \overline{1_{i}^{\prime}}}+\sum_{j=1}^{l} \varepsilon_{j} u_{\overline{2_{j}} \overline{1_{i}^{\prime}}}\right| \leq\left(\sum_{j=1}^{l} \varepsilon_{j}+\sum_{i=1}^{n} \delta_{i}\right) C .
\end{aligned}
$$

The accuracy of the result is proved.

Theorem 4 gives us information on how the approximate price behaves when $\varepsilon_{j} \rightarrow 0$ and $\delta_{i} \rightarrow 0$.

Let $X$ be securities without payment on assets dividends (for example, share, index and so on). $X$ is very often modelled as a geometric Brownian motion with constant volatility (for example, Black-Scholes option pricing model). Let us consider $X$ as a geometric Brownian motion model with multidimensional stochastic volatility. Thus, $\widetilde{\mathbb{P}}$-dynamics in $X$ are set

$$
d X_{t}=r X_{t} d t+f\left(Y_{1}, \ldots, Y_{l}, Z_{1}, \ldots, Z_{n}\right) X_{t} \widetilde{d W}_{t}^{x}, h\left(X_{t}\right)=0
$$


We calculate the approximate price of the double barrier option defined on $X$.

We write operator $\left\langle\mathfrak{L}_{2}\right\rangle$ and the density associated with $t$ and rate $m(x)$

$$
\left\langle\mathfrak{L}_{2}\right\rangle=\frac{1}{2} \bar{\sigma}^{2} x^{2} \partial_{x x}^{2}+r x \partial_{x}-r, \quad m(x)=\frac{2}{\bar{\sigma}^{2} x^{2}} \exp \left(\frac{2 r}{\bar{\sigma}^{2}} \ln x\right) .
$$

For a double barrier option with barrier value $L$ and $R$, the payoff has the form

$$
H\left(X_{t}\right) \mathbb{I}_{\tau>t}=\left(X_{t}-K\right)^{+} \mathbb{I}_{\tau>t}, I=(L, R), 0<L<K<R .
$$

To calculate the value of this parameter, we must first find the eigenvalues of operator $\left\langle\mathfrak{L}_{2}\right\rangle$ presented in (12) with boundary conditions

$$
\lim _{\mathrm{x} \rightarrow \mathrm{L}} \psi_{n}(x)=0, \quad \lim _{\mathrm{x} \rightarrow \mathrm{R}} \psi_{n}(x)=0 .
$$

Note that we have introduced regular keeling (interrupt process) boundary conditions at the ends of $L$ and $R$. The equation $-\left\langle\mathfrak{L}_{2}\right\rangle \psi_{n}=\lambda_{n} \psi_{n}, \psi_{n} \in \operatorname{dom}\left(\left\langle\mathfrak{L}_{2}\right\rangle\right)$, with boundary conditions mentioned above can be found in [11]

$$
\begin{gathered}
\psi_{n}(x)=\frac{\bar{\sigma} \sqrt{x}}{\sqrt{\ln \left(\frac{R}{L}\right)}} \exp \left(\frac{-r}{\bar{\sigma}^{2}} \ln x\right) \sin \left(\frac{n \pi \ln \left(\frac{x}{L}\right)}{\ln \left(\frac{R}{L}\right)}\right), \quad n=1,2,3, \ldots, \\
\lambda_{n}=\frac{1}{2}\left(\frac{n \pi \bar{\sigma}}{\ln \left(\frac{R}{L}\right)}\right)^{2}+\left(\frac{v^{2}}{2}+r\right), \quad v=\frac{r}{\bar{\sigma}}-\frac{\bar{\sigma}}{2} .
\end{gathered}
$$

We write the expressions for operators $\mathcal{A}_{j}$ and $\mathcal{B}_{i}$

$$
\mathcal{A}_{j}=-\vartheta_{3 j} x \partial_{x} x^{2} \partial_{x x}^{2}-\vartheta_{2 j} x^{2} \partial_{x x}^{2}, \quad \mathcal{B}_{i}=-\vartheta_{1 i} x \partial_{x}-\vartheta_{0 i} .
$$

On the basis of (5) we calculate $\mathcal{A}_{j k, n}, \mathcal{B}_{i k, n}$ and $\widetilde{\mathcal{B}}_{i k, n}$. For $k \neq n$ we obtain

$$
\begin{gathered}
\mathcal{A}_{j k, n}=-\vartheta_{3 j}\left(\frac{\left(-1+(-1)^{k+n}\right) k n\left(4 n^{2} \pi^{2} \bar{\sigma}^{4}+\left(-12 r^{2}+4 r \bar{\sigma}^{2}+\bar{\sigma}^{4}\right) \ln ^{2}\left(\frac{R}{L}\right)\right)}{2\left(k^{2}-n^{2}\right) \bar{\sigma}^{4} \ln ^{3}\left(\frac{R}{L}\right)}\right) \\
-\vartheta_{2 j}\left(\frac{\left(-1+(-1)^{k+n}\right) k n r}{\left(k^{2}-n^{2}\right) \bar{\sigma}^{2} \ln \left(\frac{R}{L}\right)}\right), \\
\mathcal{B}_{i k, n}=\vartheta_{i 1} \frac{2\left(-1+(-1)^{k+n}\right) k n}{(k-n)(k+n) \ln \left(\frac{R}{L}\right)}, \\
\widetilde{\mathcal{B}}_{i k, n}=-\vartheta_{1 i} \bar{\sigma}^{\prime}\left(Y_{k, n}\right)-\vartheta_{i 0} \bar{\sigma}^{\prime}\left(\frac{8\left(-1+(-1)^{k+n}\right) k n r \ln \left(\frac{R}{L}\right)}{\left(k^{2}-n^{2}\right)^{2} \pi^{2} \bar{\sigma}^{3}}\right), \\
v_{k, n}:=\frac{4 n k r\left(\ln (L)-(-1)^{k+n} \ln (R)\right)}{\left(k^{2}-n^{2}\right) \bar{\sigma}^{3} \ln \left(\frac{R}{L}\right)} \\
2\left(-1+(-1)^{k+n}\right) k n\left((k-n)(k+n) \pi^{2} \bar{\sigma}^{4}-2 r\left(-2 r+\bar{\sigma}^{2}\right) \ln ^{2}\left(\frac{R}{L}\right)\right) \\
\left(k^{2}-n^{2}\right)^{2} \pi^{2} \bar{\sigma}^{5} \ln \left(\frac{R}{L}\right)
\end{gathered}
$$


and for $k=n$ we obtain

$$
\begin{gathered}
\mathcal{A}_{j n, n}=-\vartheta_{3 j}\left(\frac{1}{\bar{\sigma}^{3}}\left(\frac{3 n^{2} \pi^{2} v}{\ln ^{2}\left(\frac{R}{L}\right)}-v^{3}\right)-\frac{1}{\bar{\sigma}^{2}}\left(v^{2}-\frac{n^{2} \pi^{2}}{\ln ^{2}\left(\frac{R}{L}\right)}\right)-\vartheta_{j 2}\left(\frac{1}{\bar{\sigma}^{2}}\left(v^{2}-\frac{n^{2} \pi^{2}}{\ln ^{2}\left(\frac{R}{L}\right)}\right)+\frac{v}{\bar{\sigma}}\right)\right), \\
\mathcal{B}_{i n, n}=\vartheta_{i 1}\left(\frac{2 r-\bar{\sigma}^{2}}{2 \bar{\sigma}^{2}}\right)-\vartheta_{i 0} \\
\widetilde{\mathcal{B}}_{i n, n}=-\vartheta_{i 1} \bar{\sigma}^{\prime}\left(\frac{1}{\bar{\sigma}}-\frac{r v\left(\ln ^{2}(R)-\ln ^{2}(L)\right)}{\bar{\sigma}^{4} \ln \left(\frac{R}{L}\right)}\right)-\vartheta_{i 0} \bar{\sigma}^{\prime}\left(\frac{1}{\bar{\sigma}}-\frac{r\left(\ln ^{2}(R)-\ln ^{2}(L)\right)}{\bar{\sigma}^{3} \ln \left(\frac{R}{L}\right)}\right) .
\end{gathered}
$$

The calculation of $c_{n}$ can be found in [12-14]

$$
\begin{gathered}
c_{n}=\left(\psi_{n}(x),(\mathrm{C}-K)^{+}\right)=\frac{L^{\frac{v}{\bar{\sigma}}}}{\log \left(\frac{R}{L}\right)}\left(L \Phi_{n}(v+\bar{\sigma})-K \Phi_{n}(v)\right), \\
\Phi_{n}(\gamma):=\frac{2}{\omega_{n}^{2}+z^{2}}\left(\exp (\mathfrak{K} \gamma)\left(\omega_{n} \cos \left(\omega_{n} \mathfrak{K}\right)-\gamma \sin \left(\omega_{n} \mathfrak{K}\right)-\exp (\mathfrak{U} \gamma)(-1)^{n} \omega_{n}\right),\right. \\
\omega_{n}:=\frac{n \pi}{\mathfrak{U}}, \quad \mathfrak{K}:=\frac{1}{\bar{\sigma}} \ln \left(\frac{K}{L}\right), \quad \mathfrak{U}:=\frac{1}{\bar{\sigma}} \ln \left(\frac{R}{L}\right) .
\end{gathered}
$$

The approximate option price can be calculating applying Theorems 1-3.

Note that figures are constructed component-wise in each corresponding time scale, similarly to both components in works [9] and [13].

\section{CONCLUSIONS}

This paper expands methodology of approximate pricing for a wide range of derivative assets. Derivatives payoffs can be way dependent, and the process underlying it may have a jump. Jump intensity depends on multidimensionality of volatility. We have developed a general theory of pricing derivative options which are generated by diffusion processes, where diffusion depends on two groups of variables. An algorithm for approximate price calculation is given. The price accuracy is determined. A developed theory is applied to OrnsteinUhlenbeck diffusion operator, which is expanded in eigenfunctions and eigenvalues.

The main advantage of our pricing methodology is that by combining methods of spectral theory, regular perturbation theory, and singular perturbation theory, we reduce everything to the solution of the equations to find eigenfunctions and eigenvalues.

\section{REFERENCES}

[1] McKean H.P. Elementary solutions for certain parabolic partial differential equations. Trans. Amer. Math. Soc. 1956, 82 (2), 519-548. doi: 10.1090/s0002-9947-1956-0087012-3

[2] Goldstein R.S., Keirstead W. P. On the term structure of interest rates in the presenceof reflecting and absorbing boundaries. 1997, SSRN eLibrary, 381-395.

[3] Pelsser A. Pricing double barrier options using laplace transforms. Finance Stoch. 2000, 4, 95-104.

[4] Davydov D., Linetsky V. Structuring, pricing and hedging double-barrier step options. J. Comput. Finance 2001, 5, 55-88. 
[5] Fouque J.-P., Papanicolaou G., Sircar R. Derivatives in Financial Markets with Stochastic Volatility, Cambridge University Press, 2000.

[6] Gatheral J. The Volatility Surface: a Practitioner's Guide. John Wiley and Sons, Inc. 2006.

[7] Mendoza-Arriaga R., Carr P., Linetsky V. Time-changed markov processes in unified credit-equity modeling. Math. Finance 2010, 20 (4), 527-569. doi: 10.1111/j.1467-9965.2010.00411

[8] Fouque J.-P., Jaimungal S., Lorig M. Spectral decomposition of option prices in fast mean-reverting stochastic volatility models. SIAM J. Financial Math. 2011, 2, 665-691. doi:10.1137/100803614

[9] Lorig M.J. Pricing Derivatives on Multiscale Diffusions: an Eigenfunction Expansion Approach. Math. Finance 2014, 24 (2), 331-363.

[10] Linetsky V. Spectral Methods in Derivatives Pricing. Handbooks in Operations Research and Management Science. 2007, 15, 223-299. doi: 10.1016/S0927-0507(07)15006-4

[11] Carr P., Linetsky V. A jump to default extended CEV model: An application of Bessel processes. Finance Stoch. 2006, 10 (3), 303-330. doi: 10.1007/s00780-006-0012-6

[12] Borodin A., Salminen P. Handbook of Brownian motion: facts and formulae. Birkhauser, 2002.

[13] Burtnyak I.V., Malytska A.P. Research of Ornstein-Uhlenbeck Process Using the Spectral Analysis Methods. Probl. Econ. 2014, 2, 49-56. (in Ukrainian)

[14] Fouque J.-P., Papanicolaou G., Sircar R., Solna. K. Singular perturbations in option pricing. SIAM J. Appl. Math. 2003, 63, doi:1648-1665. doi:10.1137/S0036139902401550

Received 06.11.2017

Буртняк І.В., Малицька Г. П. Застосування спектральної теорї̈ та теорї̈ збурень до дослідження процесів Орнштейна-У ленбека // Карпатські матем. публ. - 2018. - Т.10, №2. - С. 273-287.

В статті використано методи спектральної теорії та теорії сингулярних і регулярних збурень, знайдено наближену ціну двобарєрних опціонів Орнштейна-Уленбека з багатофакторною волатильністю, як розвинення за власними функціями використовуючи інфінітезимальні генератори $(l+n+1)$ вимірної дифузіі. Встановлено теорему оцінки точності наближення цін опціонів. Знайдено явні формули для знаходження вартості деривативів на основі розвинення за власними функціями та власними значеннями самоспряжених операторів з використанням крайових задач для сингулярних і регулярних збурень.

Ключові слова і фрази: спектральна теорія, сингулярна хвильова теорія, регулярна хвильова теорія, теорія Штурма-Аіувілля, інфінітезимальний генератор, багатофакторна дифузія. 